\title{
Demo: Repeatable mobile networking research with PhantomNet
}

\author{
Junguk Cho, Jonathan Duerig, Eric Eide, Binh Nguyen, Robert Ricci, \\ Aisha Syed, Jacobus Van der Merwe, Kirk Webb and Gary Wong \\ University of Utah, Salt Lake City, Utah \\ junguk.cho@utah.edu,eeide@cs.utah.edu,duerig@flux.utah.edu,binh@cs.utah.edu,ricci@cs.utah.edu \\ aisha.syed@utah.edu,kobus@cs.utah.edu,kwebb@cs.utah.edu,gtw@flux.utah.edu
}

\begin{abstract}
We will demonstrate features and capabilities of the PhantomNet testbed. PhantomNet is a mobile testbed, at the University of Utah, aimed at enabling a broad range of mobile networking related research. PhantomNet is remotely accessible and open to the mobile networking research community.
\end{abstract}

\section{CCS Concepts}

-Networks $\rightarrow$ Wireless access points, base stations and infrastructure;

\section{Keywords}

Mobile and Wireless; Testbed; Repeatable Research

\section{INTRODUCTION}

The development of various "soft" technologies, i.e., software defined networking, cloud computing, software defined radio, network function virtualization etc., are fundamentally changing the way we do networking. The increasing shift towards a mobile worldcontinued proliferation of mobile and wearable devices, growth in machine-to-machine (M2M) devices and communication and the emergence of Internet of Things (IoT), Tactile Internet and Smart Cities-suggests that future mobile and wireless networks specifically stand to benefit from exploiting these soft technologies.

To unlock this potential, educators and researchers need testbeds: facilities where the next generation of technologists can receive hands-on training on state-of-the-art equipment and where new ideas can be implemented, deployed, and rigorously evaluated under realistic but controlled conditions.

To address this need we have created the PhantomNet testbed at the University of Utah [1] (www.phantomnet.org). PhantomNet is a programable end-to-end mobile networking testbed. In PhantomNet an experimenter can instantiate a small but complete "standard" cellular network, including end-user devices, cellular base stations, compute nodes for hosting cellular core network functions, and a

\footnotetext{
${ }^{*}$ Contact author.
}

Permission to make digital or hard copies of part or all of this work for personal or classroom use is granted without fee provided that copies are not made or distributed for profit or commercial advantage and that copies bear this notice and the full citation on the first page. Copyrights for third-party components of this work must be honored. For all other uses, contact the owner/author(s).

MobiCom'16 October 03-07, 2016, New York City, NY, USA

(C) 2016 Copyright held by the owner/author(s).

ACM ISBN 978-1-4503-4226-1/16/10.

DOI: http://dx.doi.org/10.1145/2973750.2985616 network that ties the devices together. Alternatively, a researcher might use the PhantomNet building blocks to explore the feasibility of, for example, a future mobile network architecture to support IoT, or a low-latency augmented reality application, or mechanisms for dynamic spectrum allocation, etc. Multiple experimenters can use PhantomNet at the same time: each experimenter receives a separate experiment or "slice" of the testbed resources, and PhantomNet ensures that concurrent experiments will not affect each other. Below we provide an overview of the PhantomNet testbed and the features and capabilities we will be demonstrating.

\section{PhantomNet OVERVIEW}

PhantomNet is composed of three top-level components: a control framework, a set of hardware components and a set of software components. PhantomNet utilizes and builds on the Emulab control framework [3].

Hardware. In addition to traditional network testbed resources, PhantomNet provides a number of hardware resources that are of interest to researchers working with mobile platforms. PhantomNet gives access to off-the-shelf mobile handsets, or UEs (User Equipment), using mobile networking nomenclature, in the form of Android handsets. These handsets are paired with compute nodes, allowing experimenters Android Debug Bridge (ADB) access to the devices. PhantomNet also provides off-the-shelf small cell base stations, or eNodeBs according to mobile networking terminology. We use small cell eNodeBs from ip.access, operating on LTE (Long Term Evolution) spectrum bands compatible with our Android handsets.

PhantomNet also provides access to software defined radio (SDR) hardware. The SDR devices are attached as peripherals to dedicated compute nodes. When combined with the appropriate software (see below), these devices can act as base stations (eNodeBs). We have SDR hardware in the form of Ettus Research USRP B210s. These devices perform tuning, amplification, and ADC/DAC in hardware, and then communicate baseband samples over USB 3.0 links to the host nodes, allowing a great deal of flexibility in higher level signal processing.

This mix of resource types allows experimenters to target different areas of interest, from higher-level protocol interactions, down to wireless signal manipulation. To facilitate clean, repeatable experimentation, wireless devices are connected through a programmable radio frequency (RF) attenuator matrix. This matrix allows users to mix and match end-user equipment (UE devices) flexibly with access point nodes (off-the-shelf, and software-defined varieties). Relative signal strength between devices can be programmatically adjusted via the attenuator.

Software. Software resources available in PhantomNet include: an evolved packet core suite called OpenEPC (www.openepc.com), 


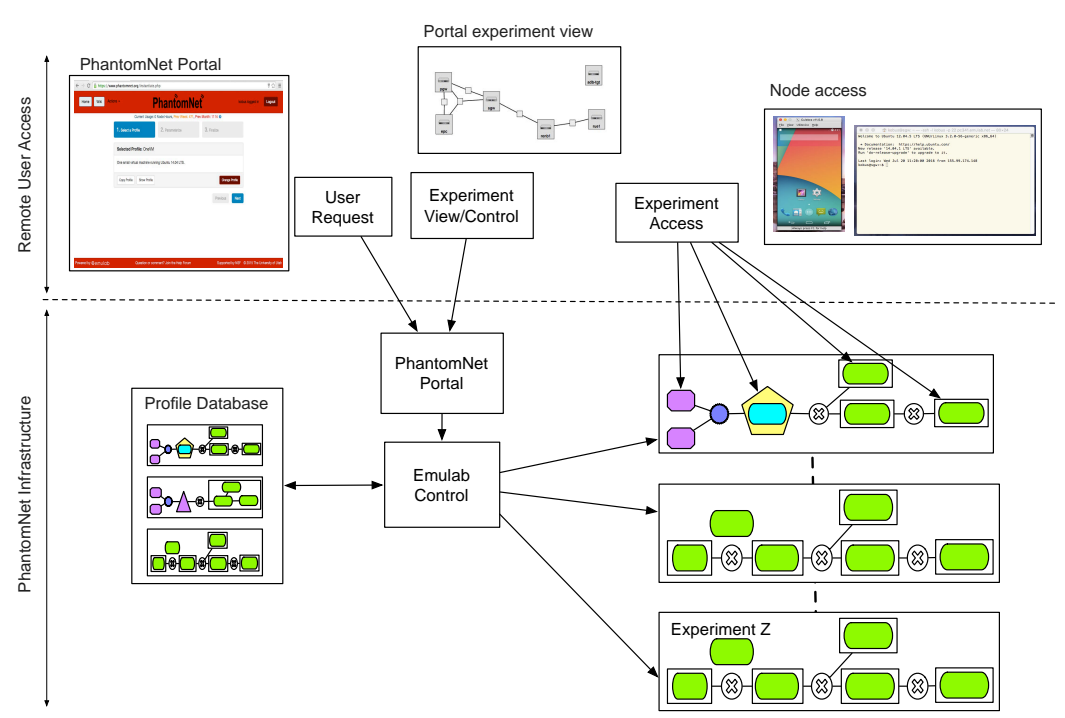

Figure 1: PhantomNet workflow

and radio access network (RAN) over SDR implementations from OpenAirInterface (OAI) (www.openairinterface.org) and OpenLTE (openlte.sourceforge.net). OpenEPC is an Evolved Packet Core (EPC) software stack which includes much of the functionality codified by the 3GPP LTE version 12 specification. This includes services for handling end-user device (UE) attachment, handover (mobility), policy and charging, etc. The OAI software includes SDR-based user equipment (UE) and access point (eNodeB) implementations, and an emerging 3GPP LTE Evolved Packet Core (EPC) implementation. OpenLTE is similar, but is built on top of GNU Radio and does not tread much beyond the radio access layers of LTE. PhantomNet allows users to request these resources and configure them for particular purposes.

\section{DEMONSTRATION OVERVIEW}

We will demonstrate a number of PhantomNet features and capabilities including:

Experimental workflow. We will demonstrate the basic PhantomNet workflow depicted in Figure 1 Specifically we will demonstrate how users can:

- Access the PhantomNet portal and select a "profile", from the PhantomNet profile database to instantiate an experiment. A profile is a complete description of an experiment and encapsulates all resources and dependencies associated with an experiment.

- View and control their experiments in PhantomNet. This includes general experimental functions like determining the state of nodes in the experiment, rebooting nodes in the experiment, terminating or extending the lifetime of the experiment etc. PhantomNet also allows programmable per-experiment control of the radio frequency links (in the RF attenuator matrix) between RF equipment.

- Access the nodes in their experiment. This includes access to nodes via an embedded browser shell, via direct terminal (ssh) access to a node, as well as Android Debug Bridge (ADB) access to Android devices in the experiment.

Example experiments. We will demonstrate some of the existing experiment profiles available in PhantomNet, including:

- Off-the-shelf (OTS) $4 G$ cellular network. This profile combines OTS hardware (Android smartphone and small-cell base station) with an evolved packet core (EPC) core mobile network to realize a "complete" 4G mobile network.

- Emulated $4 G$ with open source. This profile makes use of an open source mobile software stack (OAI) to realize the basic components of a $4 \mathrm{G}$ mobile network.

- Service control platfrom for a mobile software defined infrastructure. This profile shows how PhantomNet was used to create a service control platform to enable service evolution in a mobile SDI [2].

- Open source on SDR with OTS hardware. This profile shows how an open source mobile stack (OAI) can be combined with PhantomNet's SDR and OTS hardware (Android smartphone) to realize an LTE RAN.

Repeatable experimentation. We will demonstrate PhantomNet features that enable repeatable experimentation:

- Creating your own profile. A typical experimental workflow might involve starting with a profile "close" to the desired experiment, modifying the experiment as needed and creating a new profile that captures the changes.

- Scripted interaction with OTS devices. PhantomNet's ADB access to OTS UEs allows for standard Android tools to be used in the testbed to enable scripted control of these devices.

\section{ACKNOWLEDGMENTS}

This material is based upon work supported by the National Science Foundation under grant number 1305384.

\section{REFERENCES}

[1] A. Banerjee, J. Cho, E. Eide, J. Duerig, B. Nguyen, R. Ricci, J. Van der Merwe, K. Webb, and G. Wong. Phantomnet: Research infrastructure for mobile networking, cloud computing and software-defined networking. GetMobile: Mobile Computing and Communications, 19(2):28-33, 2015.

[2] A. Syed and J. Van der Merwe. Proteus: A network service control platform for service evolution in a mobile software defined infrastructure. In MobiCom, Oct 2016.

[3] B. White, J. Lepreau, L. Stoller, R. Ricci, S. Guruprasad, M. Newbold, M. Hibler, C. Barb, and A. Joglekar. An Integrated Experimental Environment for Distributed Systems and Networks. In OSDI, Dec. 2002. 